\title{
Breast Cancer in Males (BCM), Does It Really Differ? National Cancer Institute Experience (NCI), Cairo University, Egypt
}

\author{
Ahmed El Sayed Fathalla, Marwa Nabil Abd El Hafez \\ National Cancer Institute, Cairo University, Giza, Egypt \\ Email:drasf1975@gmail.com, Marwa_nabil_25@yahoo.com
}

Received 3 April 2016; accepted 10 May 2016; published 13 May 2016

Copyright (C) 2016 by authors and Scientific Research Publishing Inc.

This work is licensed under the Creative Commons Attribution International License (CC BY). http://creativecommons.org/licenses/by/4.0/

(c) (i) Open Access

\section{Abstract}

Background: Carcinoma of the male breast is relatively a rare disease accounting for about $1 \%$ of all cancer in men. Its treatments are based mainly on those of Female Breast Cancer (BCF). Materials and Methods: This is a single institution retrospective study including all presenting to the NCI, Cairo University with Breast Cancer in a Male (BCM) in the last 11 years between Jan. 2005 until Jan. 2016. Data were collected from patient's files from the statistical department then analyzed. Aims: To study the clinico-pathological characteristics, the presentation, workup, surgical approaches and postoperative complications and outcome of management, with addressing similarities and difference from BCF. Results: 64 patients were included in this cohort. The mean age was 58.6 \& the median age was 59 years (range: 31 - 87 years). The main presenting symptomatology was retroareolar breast lump (50 cases, 78.1\%). Most of our cases were advanced; 22 cases (34.3\%) were stage III \& 16 cases (25\%) were stage IV. Surgery was performed for 50 patients; MRM for 26 cases $(40.6 \%)$, RM for 18 cases $(28.1 \%) \&$ toilet mastectomy for 6 cases $(9.3 \%)$. Primary closure was feasible in 34 patients $(68 \%)$ while 16 cases $(32 \%)$ required reconstruction by local or pedicled flaps. Tamoxifen is the most important non-surgical treatment. It was given to all our cases (64 cases, $100 \%$ ) either postoperatively as adjuvant management ( 36 cases, $56.2 \%$ ) or as palliation for metastatic disease $(28$ cases, $43.8 \%)$. Chemotherapy was given to 32 patients $(100 \%)$ as an adjuvant for 24 cases $(75 \%)$ and as a palliation for 8 cases $(25 \%)$. Radiotherapy was given to 26 patients $(100 \%)$ as adjuvant therapy for 20 cases $(76.9 \%)$ and as a palliation for 6 cases $(\mathbf{2 3 . 1 \%})$. The only significant factor determining the overall 5 years survival was the stage of the disease. LN status \& surgery type were of border line significance (better survival with negative LN \& with MRM). The 5-year Overall Survival (OS) \& Disease Free Survival (DFS) for the whole group were $66 \%$ \& $52 \%$ respectively. Within the DFS there was no significant variable; however, the stage and type of surgery were of borderline significance, with better survival with early stage disease (I \& II) and with MRM (both were 61\%). Conclusion: BCM has many similarities to BCF, but it harbours many different genetic and pathologic features. They obtain similar prognostic factors 
and similar stage-for-stage survival. They are always advanced to $\mathrm{T} 4$ stage rapidly due to the lack of breast parenchyma with higher ER expression in BCM than BCF patients.

\title{
Keywords
}

\author{
Male Breast Cancer, Clinicopathological, Females, Outcome
}

\section{Introduction}

This rare disease accounts for about $1 \%$ of all cancer in men and $1 \%$ of all breast cancer worldwide. It is responsible for $0.15 \%$ of cancer deaths in males. The global distribution of BCM is similar to that of BCF (rare in areas of low incidence of breast cancer). However, in Egypt, an area with relatively low BCF incidence, BCM is common. This is due to the high rate of cirrhosis and its related consequence of liver cell failure with resultant hormonal imbalance (estrogen/androgen imbalance) [1] [2].

This is a disease of old age (above 60), although it was reported from 5 to 93 years with an incidence increasing progressively with age. It is hormonally driven; main risk factors include estrogen/androgen imbalance \& positive family history of BCF (especially first degree relatives \& young age at diagnosis) (15\% - 20\% of BCM patients have positive family history of BCF). Other factors are: prolonged heat exposure, chest wall irradiation, relative hyperestrogeny (mumps orchitis, infectious orchitis, undescended testes, orchiectomy, gynecomastia, exogenous estrogen intake, obesity, liver disease and Klinefelter syndrome (increases the risk 50-folds\}). BRCA2 mutations are major predisposing factors to BCM while BRCA1 mutations were not associated with a significant risk of breast cancer [3].

85\% of patients present by a painless sub-areolar mass with rare involvement of the nipple \& slight predilection for the left side (left/right ratio is 1.07/1). Nipple discharge in females is usually non bloody and associated with benign lesions. $20 \%$ - 30\% of patients presenting with pain or bloody discharge have $80 \%$ likelihood of an underlying tumor, including ductal carcinoma insitu (DCIS). Discharge cytology is often diagnostic in these cases [4].

Open biopsy, although expensive, remains the gold standard for BCM diagnosis. Fine Needle Aspiration Cytology (FNAC) based diagnosis can safely avoid open biopsy. Combined Clinical Examination (CE) and FNAC can diagnose most of patients with mammography recommended only when FNA \& CE indicate a positive disease. Mammogram gives no additional data to the already provided by CE \& FNAC nor does it change the management of any. Nearly all the histological sub-types of BCF are reported in men [5]-[7].

Historically, most men were treated with Radical Mastectomy (RM) which gave equivalent survival \& local recurrence rates as Modified Radical Mastectomy (MRM) which is the recommended surgical procedure with Total Mastectomy (TM) only reserved for smaller and DCIS lesions. Obviously, segmental (conservative) mastectomy does not have a role in males [8].

Two-level axillary dissection remains the gold standard for pathologic staging of the clinically negative axilla. Sentinel Lymph Node Biopsy (SLNB) can also be used in BCM as BCF to decrease the magnitude and morbidity of breast surgery in males. Also DCIS in males could be treated with TM rather than MRM and theoretically, one of the current surgical recommendations for DCIS in females could be extended to males. Men may have higher risk of internal mammary nodal disease where they may benefit more from internal mammary radiation [9].

The main problem in reconstruction of male breast is tissue deficiency following surgery. It is often impossible to close wounds primarily without tension, resulting in edges dehiscence with delayed healing. In early stages, thoracic flaps are used based on the superficial thoracic artery, or thoraco-epigastric fasciocutaneous flaps based on the intercostal perforators. When large deep excisions are required (radiation therapy is mandatory), myocutaneus flaps, such as latissimus dorsi (LDMCF) or transverse rectus abdominis (TRAM) are performed. When rib resection is required, myocutaneus flaps are used with alloplastic materials (stainless steel wires \& Marelex meshes) or the so called cyano-methyl-methacrylate (bone cement) sandwich [10].

Adjuvant hormonal therapy and chemotherapy, using the same guidelines for women, are recommended for men. Tamoxifen is the primary therapy for metastatic disease with chemotherapy reserved for hormone-refrac- 
tory disease. BCM have higher rate of hormone receptor positivity (ER \& PR) than do BCF. 80\% are ER positive $\& 75 \%$ are PR positive. Tamoxifen improves survival rates of all hormone receptor positive tumors given for periods not less than five years [11].

Men are less likely to receive chemotherapy after surgery than their matched females. Similar to BCF, adjuvant chemotherapy decreases distant relapse rates for node positive patients and also become beneficial for node negative disease. Downsizing tumors using neoadjuvant chemotherapy and subsequent salvage mastectomy or palliative chest wall radiation are strategies for locally advanced disease [12].

Metastatic disease has always been treated by hormonal therapy with overall response rates of $70 \%$ for receptor positive tumors. ER positive tumors appear to respond to hormonal manipulations compared to ER negative tumors. Tamoxifen is the first line hormonal therapy because of its efficacy and limited toxicity. Despite its efficacy, Tamoxifen was discontinued in $20 \%$ of cases due to its side effects (decreased libido, deep-vein thrombosis, mood alterations, hot flashes and depression) [13].

Patients with metastatic disease who relapsed on Tamoxifen are treated with second-line hormonal therapy (aromatase inhibitors) (similar to the situation for postmenopausal BCF patients). Palliative chemotherapy is reserved for non-responders and receptor-negative tumors with $40 \%$ response for all regimens [14].

Although BCM tends to present at later stages than do BCF (low index of suspicion \& lack of male screening), the discrepancy in stage distribution, and the difference in overall prognosis between them is shrinking. As females; BCM carries the same prognostic factor with stage-for-stage outcomes almost the same. Axillary lymph node status, tumor size, histologic grade and hormone receptor status are the most significant factors affecting prognosis. By far, nodal disease is the most significant negative prognostic factor affecting survival with the five-year overall survival rates for all stages range from $36 \%$ to $66 \%$ [15].

This cohort aims to study the clinico-pathological features of BCM and to determine the clinical presentation, workup, outcome of management, with addressing similarities and difference from BCF.

\section{Materials and Methods}

This is a retrospective non-randomized single institutional study of 64 patients with BCM treated at the National Cancer Institute, Cairo University, Egypt during the last 11 years between Jan. 2005 \& Jan. 2016. Data were collected from patient's files from the statistical department then analyzed. Each Patient was revised for age, family history, clinical presentation, stage, investigations, surgery, postoperative complications, radiotherapy or chemotherapy given and state at last follow-up. Outcome analysis was done including OS and DFS \& the patterns of treatment failure were classified into local recurrence and distant metastasis.

Ethical clearance for the conduction of this study was obtained from our institute ethical committee.

\section{Results}

\subsection{Clinical Picture}

This cohort included 64 patients with BCM. The mean age was 58.6 years (median was 59 years with range 31 87 years). Most of our cases present by breast lump (50 cases, 78.1\%) occupying the retroareolar region (46 cases, $71.9 \%$ ) with mild predilection to the left side (38 cases, 59.4\%). Patient characteristic in our study is presented in Table 1.

The mean delay between the complaint and presentation was 11.5 months (median was 11 months \& range: 2 - 37 with SD: 7.71). The median time between diagnosis and definitive surgery was one month (range: 0 - 48 months). This marked delay in some patients is due to the patient hesitation with primary refusal of surgery.

\subsection{Investigation}

The majority of our patients underwent a form of tissue diagnosis mainly in the form of incisional biopsy (28 cases, 43.7\%). As ER, PR \& HER-2 neu status should be evaluated in all patients, tissue biopsy was preferred rather than FNAC. Other diagnostic modalities used in this cohort are shown in Table 2.

\subsection{Treatment}

Mastectomy was done in 50 cases. Most of our cases were treated by MRM (26 cases, 40.6\%), RM was done in (18 cases, 28.1\%), total (simple) mastectomy was performed for (6 patients, 9.3\%) with distant deposits, marked 
tumor ulceration and fungation. 4 patients (6.2\%) refused surgery and lost follow up shortly after biopsy. 12 cases $(9.4 \%)$ proved to have distant deposits and were treated with palliative hormonal therapy. Primary closure was feasible in (34 cases, 68\%). Local flaps were needed in 8 cases (16\%), while LD myocutaneus flap was done for 6 cases $(12 \%)$. In only 2 patients $(4 \%)$ chest wall resection of 3 ribs was needed \& reconstruction was done using bone cement $\&$ double layer proline mesh covered by TRAM myocutaneus flap (Figure 1, Figure 2). Different surgical \& non surgical procedures done in this study with the different reconstructive procedures used are presented in Table 2.

Table 1. Patient characteristics in our cohort.

\begin{tabular}{|c|c|}
\hline $\begin{aligned} \text { Age: } & \\
\bullet & <50 \text { years } \\
\bullet & >50 \text { years }\end{aligned}$ & $\begin{array}{l}18(28.2 \%) \\
46(71.8 \%)\end{array}$ \\
\hline $\begin{aligned} & \text { Family history: } \\
& \text { - } \text { Negative } \\
& \text { - } \text { Positive }\end{aligned}$ & $\begin{array}{l}58(15.6 \%) \\
6(9.3 \%)\end{array}$ \\
\hline $\begin{array}{l}\text { Symptomatology: } \\
\text { - Lump } \\
\text { - Ulcer } \\
\text { - Gynecomastia } \\
\text { - Bony aches }\end{array}$ & $\begin{array}{l}50(78.1 \%) \\
8(12.5 \%) \\
4(6.3 \%) \\
2(3.1 \%)\end{array}$ \\
\hline $\begin{aligned} \text { Site: } & \\
\text { - } & \text { Retroareolar } \\
\text { - } & \text { Upper outer quadrant }\end{aligned}$ & $\begin{array}{l}46(71.9 \%) \\
18(28.1 \%)\end{array}$ \\
\hline $\begin{array}{c}\text { Side \& Bilaterality: } \\
\text { - } \text { Bilateral } \\
\text { unilateral } \\
\text { - Right } \\
\text { Left }\end{array}$ & $\begin{array}{l}4(6.2 \%) \\
60(93.8 \%) \\
26(40.6 \%) \\
38(59.4 \%)\end{array}$ \\
\hline $\begin{array}{l}\text { HER-2: } \\
\text { - } \text { Positive } \\
\text { - Negative }\end{array}$ & $\begin{array}{l}16(25 \%) \\
48(75 \%)\end{array}$ \\
\hline $\begin{array}{ll}\text { Molecular subtypes: } \\
\text { - } & \text { Luminal A } \\
\text { - } & \text { Luminal B } \\
\text { - } & \text { HER2 overexpression } \\
\text { - } & \text { Basal like (Triple negative) }\end{array}$ & $\begin{array}{l}48(75 \%) \\
12(19 \%) \\
4(6 \%) \\
0\end{array}$ \\
\hline $\begin{array}{r}\text { T-stage: } \\
-\mathrm{T} 1 \\
-\mathrm{T} 2 \\
-\mathrm{T} 3 \\
-\mathrm{T} 4\end{array}$ & $\begin{array}{l}4(6.2 \%) \\
28(43.7 \%) \\
8(12.5 \%) \\
24(37.5 \%)\end{array}$ \\
\hline $\begin{array}{ll}\text { N-stage: } \\
\text { - } \\
\text { - } \mathrm{pN} 0 \\
\text { - } \mathrm{pN} 1 \\
\text { - } \\
\text { Missing }\end{array}$ & $\begin{array}{l}22(34.3 \%) \\
18(28.1 \%) \\
4(6.2 \%) \\
20(31.2 \%)\end{array}$ \\
\hline $\begin{array}{r}\text { M-stage: } \\
-\quad \text { M0 } \\
\text { - } \text { M1 }\end{array}$ & $\begin{array}{l}50(78.1 \%) \\
16(25 \%)\end{array}$ \\
\hline 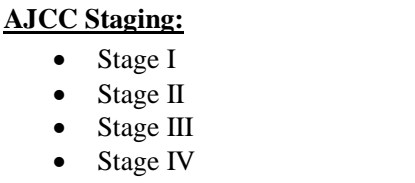 & $\begin{array}{l}4(6.2 \%) \\
22(34.3 \%) \\
22(34.3 \%) \\
16(25 \%)\end{array}$ \\
\hline
\end{tabular}


Table 2. Diagnostic methods, surgical \& non surgical procedures, postoperative complications and different reconstructive method.

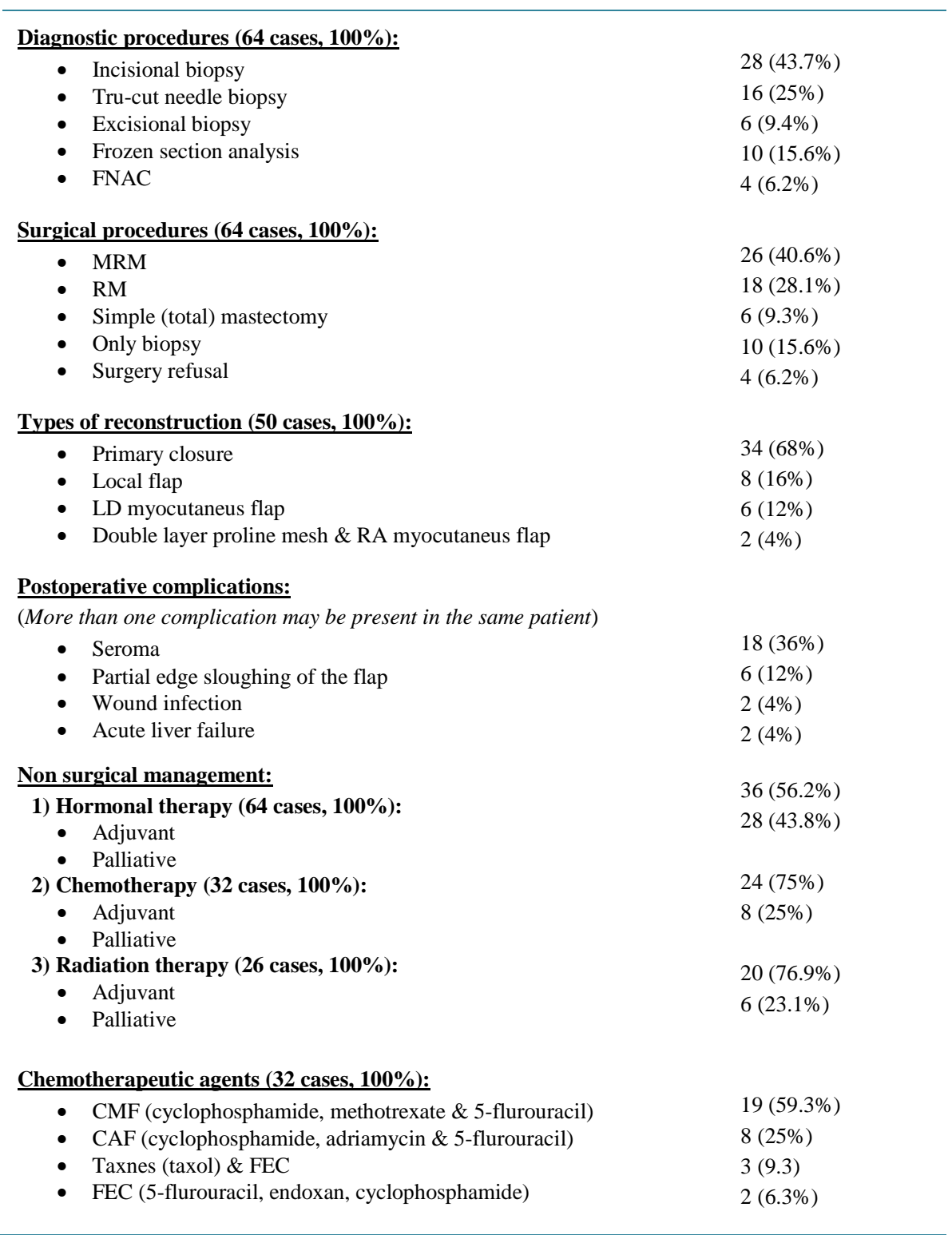
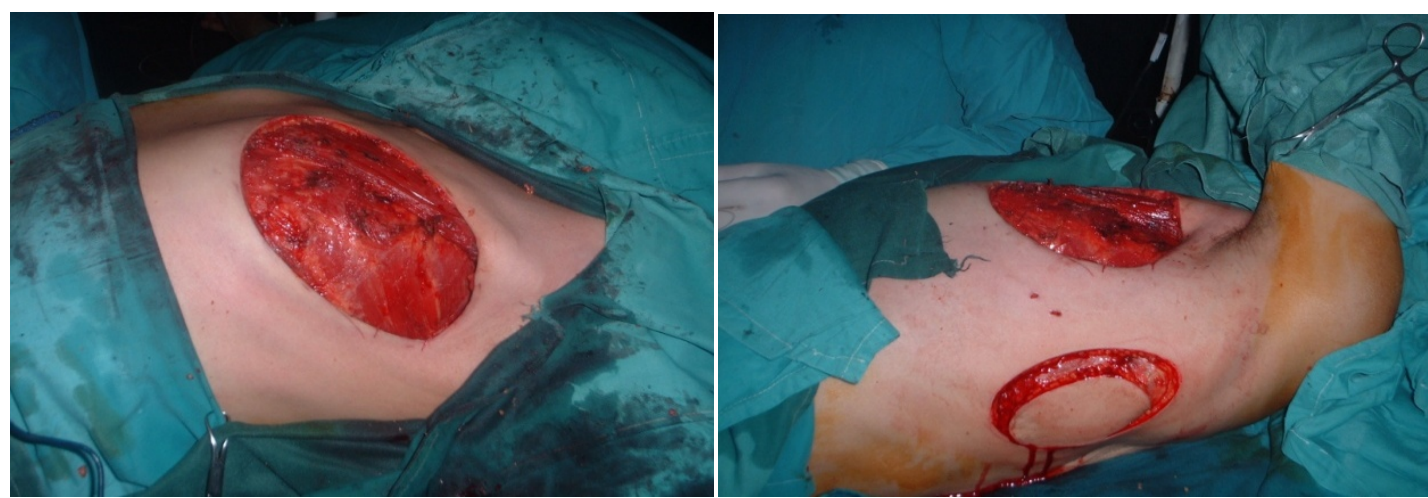

Figure 1. Postoperative defect after mastectomy (left) with reconstruction by LD myocutaneous flap (right). 

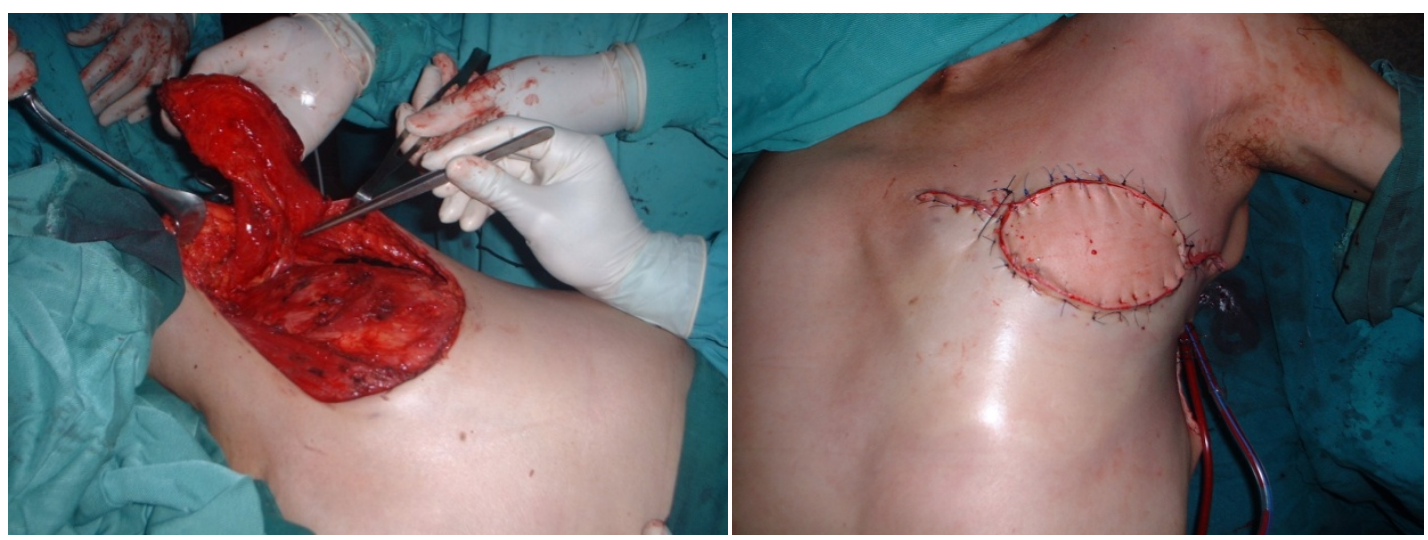

Figure 2. Elevation of the LD myocutaneous flap (left) and its rotation for closure of a large skin defect (right).

Histopathological examination of our tumors revealed that all our cases (64 patients, 100\%) were IDC and nearly all patients (60 cases, 93.7\%) were intermediate grade tumors (grade II), while only 4 cases (6.3\%) were high grade tumors (grade III).

44 patients underwent definitive treatment with axillary clearance. Half of them (22 patients, 50\%) proved to harbour positive nodal disease. The mediantotal dissected nodes was 26 nodes (mean: 13.18, range: 6 - 21 with SD: 4.46) while the median for positive axillary nodes was 12 nodes (mean: 2.32, range: 3 - 11 with SD: 3.39). Capsular invasion was noticed in all cases with positive axillary nodes (22 cases, 50\%). ER \& PR was done in 36 patients only $(81.8 \%)$ where 30 cases (68.1\%) showed strong ER \& PR positivity. HER-2 neu assessment was done to all cases and it showed over expression in only 16 cases (25\%).

Of the 44 patients (100\%) who underwent curative surgery, local recurrence occurred in 6 cases, $13.6 \%$ ) while 14 patients (31.8\%) developed systemic deposits during the course of the disease even after an apparently good local control by surgery with. 2 of our cases (4.5\%) suffered both local \& systemic failure. At the end of our study (of the 44 patients who underwent curative surgery), 24 cases (54.5\%) were alive free of the disease. 4 cases $(9.1 \%)$ were alive with local failure. Another 4 cases $(9.1 \%)$ were alive with distant failure. 2 patients (4.6\%) were alive with both local \& distant failure. And finally 10 patients (22.7\%) were dead.

Different variables were analyzed (age, stage, side, site, LN status \& type of surgery) to determine specific variables affecting the 5 years OS \& DFS. As regards the 5 years OS, the only significant variables by univariate analysis were stage $\&$ side. There were 2 factors with borderline significance; LN positivity with better survival with negative LN cases and surgery type with better survival with MRM. Multivariate analysis was done using Cox-regression test for the significant factors affecting survival on univariate analysis. Only the stage was significant (p-value; 0.041). The overall 5 years survival for the whole group was 66\%. After exclusion of the 16 cases with distant deposits from the start, the 5 years DFS for the whole group was 52\%. Within the DFS there was no significant variable, however, the stage and type of surgery were of borderline significance, with better survival with early stage disease (I \& II) and with MRM (both were 61\%).

\section{Discussion}

BCM is a rare disease accounting for $1 \%$ of all cases of cancer in men. The median age at diagnosis in most studies was 68 years. In this cohort most of our patients were above 50 y with a peak age around 60 years. $78 \%$ of our patients presented by breast lump. $72 \%$ of these lumps were retroareolar with slight predilection to the left side (60\%) [2] [16].

It tends to present at a later stages than BCF, probably due to low index of suspicion and lack of male screening. In our cohort the mean delay between complaint and presentation was 11.5 months. This delay resulted in more advanced stages at presentation. 22 cases was stage II (34.3\%, another 22 cases (34.3\%) was stage III, 16 cases (25\%) with stage IV and only 4 cases (6.2\%) were stage I [17].

Surgery is the mainstay for resectable BCM. RM was traditionally the usual choice. This trend is no more accepted where surgery evolved over the past 2 decades towards MRM and even TM for smaller \& DCIS lesions. In this work 50 patients underwent mastectomy. Most of them were treated by MRM (26 cases, 40.6\%) (Figure 1), RM was done in (18 cases, 28.1\%), total (simple) mastectomy was performed for (6 patients, 9.3\%) with 
distant deposits, marked tumor ulceration and fungation. 4 patients (6.2\%) refused surgery and lost follow up shortly after biopsy. 12 cases (9.4\%) proved to have distant deposits and were treated with palliative hormonal therapy [8] [18].

Most of our cases after mastectomy were closed primarily (34 cases, 68\%). In 16 cases (32\%), reconstruction by flaps was needed. Local flaps were needed in 8 cases (16\%), while LD myocutaneus flap was done for 6 cases (12\%) (Figure 1, Figure 2). In only 2 patients (4\%) chest wall resection of 3 ribs was needed \& reconstruction was done using bone cement $\&$ double layer proline mesh covered by TRAM myocutaneus flap [10] [19].

Because the male breast contains only ductal tissue, most BCM are of ductal type and nearly $90 \%$ are invasive with the remaining $10 \%$ are non-invasive carcinomas. $80 \%$ of tumors are invasive duct carcinoma; lobular carcinomas are less common than in females and represents only $1 \%$ of cases. In men with Klinefelter syndrome the risk of BCM approaches that of females; as these men actually develop hypertrophied breasts that contain both acini and lobules. Non invasive carcinomas in men are all low (grade I) to intermediate grade (grade II) DCIS with LCIS being extremely rare. The histopathology of our tumors showed that all our patients (64 cases, $100 \%$ ) were IDC and almost all cases (60 cases, 93.7\%) were of the intermediate grade (grade II) with only 4 cases were high grade (grade III) tumors [6] [7].

Most of cases of BCM are ER positive (65\% - 94\%) in most studies; therefore a greater percentage of male patients will be treated with Tamoxifen or will respond to hormonal manipulation than do the female patients. Similarly BCM is more commonly PR positive (93\% in one study). In our cohort ER \& PR examination was done in 36 cases (100\%) where ER was positive in 30 cases (83.3\%) and PR was positive in 28 cases (77.7\%) [11] [20].

Surgery with a curative intent should always be the aim. In this work curative surgery was done in 44 cases (68.7\%), palliative surgery was done for 6 cases (9.4\%). Hormonal therapy was given to all patients (64 cases, $100 \%$ ) either postoperative as adjuvant therapy (36 cases, 56.2\%) or in metastatic cases as a palliation (28 cases, 43.8\%). CTH was given to 32 patients (50\%) as adjuvant treatment (24 cases, 37.5\%) and as a palliation in (8 cases, $12.5 \%)$. Radiotherapy was given to 26 cases (59.3\%) as adjuvant therapy (20 cases, 31.3\%) and asa palliation in (6 cases, 9.3\%) [8] [9].

Cutuli, 2007 reported a 10\% to 30\% over expression in the HER-2 neu. In our work HER-2 neu was over expressed only in $25 \%$ of cases. Trastuzumab is a monoclonal antibody against to HER-2 receptor, but its role in BCM is still unknown. Hayashi et al., 2009 studied paclitaxel plus trastuzumab therapy and achieved a good response in both the primary and metastatic sites. Although no randomized study exists, trastuzumab therapy is recommended in HER2-positive patients. It was given in this work to all positive HER2 neu cases achieving better response than those HER2 negative patients in primary and metastatic regions [8] [21] [22].

The most significant prognostic factors for BCM are AJCC staging system particularly tumor size and LN status. Tumor size greater than $3 \mathrm{~cm}$ significantly impaired prognosis. The 5 years survival was directly related to the number of nodes involved, 55\% when 4 or more nodes were positive, $73 \%$ for 1 to 3 positive nodes, and $90 \%$ for node negative patients ( $84 \%$ at 10 years). Skin involvement, chest wall fixation, and tumor ulceration (all are common in BCM than BCF) were not independent prognostic factors As regard 5 years OS in our cohort, the only significant variables by univariate analysis were stage and side. There were 2 factors with borderline significance, LN positivity with a better survival with negative LN cases (88\%) and type of surgery with better survival with MRM (89\%). The latter is explained by the fact that RM was usually performed for more locally advanced tumors [23].

\section{Conclusion}

BCM is not a biologically more aggressive disease than the same condition in females. BCM should be treated with curative intent in most cases. Similar to the situation in females, such treatment should include optimal (but not overly aggressive) local control, adjuvant tamoxifen for receptor-positive tumors (most tumors in men) and adjuvant chemotherapy for high-risk patients.

\section{References}

[1] Dabakuyo, T.S., Dialla, O., Gentil, J., et al. (2012) Breast Cancer in Men in Cote d’Or (France): Epidemiological Characteristics, Treatments and Prognostic Factors. European Journal of Cancer Care (England), 21, 809-816. http://dx.doi.org/10.1111/j.1365-2354.2012.01365.x 
[2] Weiss, J., Moysich, K. and Swede, H. (2005) Epidemiology of Male Breast Cancer. Cancer, 14, 20-26.

[3] Lee, S. and Ellis, R. (2004) Male Breast Cancer. Journal of the National Cancer Institute, 96, 338-339. http://dx.doi.org/10.1093/jnci/djh062

[4] Jackson, R. (2003) Breast Cancer in Men. Annals of Internal Medicine, 139, 300-305. http://dx.doi.org/10.7326/0003-4819-139-4-200308190-00022

[5] Adibelli, Z.H., Oztekin, O., Postaci, H. and Uslu, A. (2009) The Diagnostic Accuracy of Mammography and Ultrasound in the Evaluation of Male Breast Disease: A New Algorithm. Breast Care, 4, 255-259. http://dx.doi.org/10.1159/000226284

[6] Adibelli, Z.H., Oztekin, O., Gunhan-Bilgen, I., et al. (2010) Imaging Characteristics of Male Breast Disease. Breast Journal, 16, 510-518. http://dx.doi.org/10.1111/j.1524-4741.2010.00951.x

[7] Ozmen, V. (2014) Breast Cancer in Turkey: Clinical and Histopathological Characteristics (Analysis of 13.240 Patients). Journal of Breast Health, 10, 98-105. http://dx.doi.org/10.5152/tjbh.2014.1988

[8] Patten, D.K., Sharifi, L.K. and Fazel, M. (2013) New Approaches in the Management of Male Breast Cancer. Clin Breast Cancer, 13, 309-314. http://dx.doi.org/10.1016/j.clbc.2013.04.003

[9] Chakravarthy, A. and Kim, C.R. (2002) Post-Mastectomy Radiation in Male Breast Cancer. Radiotherapy and Oncology, 65, 99-103. http://dx.doi.org/10.1016/S0167-8140(02)00210-4

[10] Zygogianni, A.G., Kyrgias, G., Gennatas, C., et al. (2012) Male Breast Carcinoma: Epidemiology, Risk Factors and Current Therapeutic Approaches. Asian Pacific Journal of Cancer Prevention, 13, 15-19. http://dx.doi.org/10.7314/APJCP.2012.13.1.015

[11] Perkins, G. and Middleton, L. (2003) Breast Cancer in Men. BMJ, 327, 239-240. http://dx.doi.org/10.1136/bmj.327.7409.239

[12] Hayes, T.G. (2009) Pharmacologic Treatment of Male Breast Cancer. Expert Opinion on Pharmacotherapy, 10, 24992510. http://dx.doi.org/10.1517/14656560903200634

[13] Gomez-Raposo, C., Zambrana Tevar, F., Sereno Moyano, M., Lopez Gomez, M. and Casado, E. (2010) Male Breast Cancer. Cancer Treatment Reviews, 36, 451-457. http://dx.doi.org/10.1016/j.ctrv.2010.02.002

[14] Fogh, S., Hirsch, A.E., Langmead, J.P., et al. (2011) Use of Tamoxifen with Postsurgical Irradiation May Improve Survival in Estrogen and Progesterone Receptor-Positive Male Breast Cancer. Clinical Breast Cancer, 11, 39-45. http://dx.doi.org/10.3816/CBC.2011.n.007

[15] Clark, J.L., Nguyen, P.L., Jaszcz, W.B., Jatoi, A. and Niehans, G.A. (2000) Prognostic Variables in Male Breast Cancer. American Surgeon, 66, 502-511.

[16] Cutuli, B. (2007) Strategies in Treating Male Breast Cancer. Expert Opinion on Pharmacotherapy, 8, 193-202. http://dx.doi.org/10.1517/14656566.8.2.193

[17] Fentiman, I.S., Fourquet, A. and Hortobagyi, G.N. (2006) Male Breast Cancer. Lancet, 367, 595-604. http://dx.doi.org/10.1016/S0140-6736(06)68226-3

[18] Giordano, S.H. (2005) A Review of the Diagnosis and Management of Male Breast Cancer. Oncologist, 10, 471-479. http://dx.doi.org/10.1634/theoncologist.10-7-471

[19] Reis, L.O., Dias, F.G., Castro, M.A. and Ferreira, U. (2011) Male Breast Cancer. Aging Male, 14, 99-109. http://dx.doi.org/10.3109/13685538.2010.535048

[20] Fentiman, I.S. (2009) Male Breast Cancer: A Review. Ecancermedicalscience, 3, 140. http://dx.doi.org/10.3332/ecancer.2009.140

[21] Selcukbiricik, F., Tural, D., Aydogan, F., et al. (2013) Male Breast Cancer: 37-Year Data Study at a Single Experience Center in Turkey. Journal of Breast Cancer, 16, 60-65. http://dx.doi.org/10.4048/jbc.2013.16.1.60

[22] Hayashi, H., Kimura, M., Yoshimoto, N., et al. (2009) A Case of HER2-Positive Male Breast Cancer with Lung Metastases Showing a Good Response to Trastuzumab and Paclitaxel Treatment. Breast Cancer, 16, 136-140. http://dx.doi.org/10.1007/s12282-008-0060-1

[23] Arslan, U.Y., Oksüzoglu, B., Ozdemir, N., et al. (2012) Outcome of Non-Metastatic Male Breast Cancer: 118 Patients. Medical Oncology, 29, 554-560. http://dx.doi.org/10.1007/s12032-011-9978-9 